\title{
Kurzfristige Wirtschaftlichkeit als Leitmaxime
}

\author{
Die Verschreibungs-Limitatio der neuen Hepatitis-C-Proteaseinhibitoren durch \\ das BAG verfehlt ihre Ziele und ist kontraproduktiv.
}

\section{Philip Bruggmann}

Chefarzt Innere Medizin, Arud Zentren für Suchtmedizin

Korrespondenz:

Dr. med. Philip Bruggmann

Arud Zentren für Suchtmedizin

Konradstrasse 32

CH-8005 Zürich

p.bruggmann[at]arud.ch

\section{Hintergrund}

In der Schweiz leiden ca. 70000 Personen an einer chronischen Hepatitis C. Mehr als die Hälfte hat sich durch Drogenkonsum angesteckt. Die Prävalenz unter Drogenkonsumierenden beläuft sich in der Schweiz auf $42 \%$. Die Hepatitis-C-bedingte Mortalität übersteigt inzwischen diejenige von HIV und nimmt nach dem 45. Lebensjahr exponentiell zu $[1,2]$. Bei zusätzlichen leberbelastenden Faktoren (Alkohol, Cannabis, Medikamente usw.) steigt die Gefahr der Fibrosierung und letztendlich eines Leberversagens oder eines hepatozellulären Karzinoms [3]. Leberversagen ist inzwischen die Haupttodesursache bei Drogenpatienten, noch vor Überdosis (unveröffentliche Daten, Arud, 2010). Hepatitis-CTherapien bei aktiv Drogenkonsumierenden erwiesen sich als kosteneffizient [4].

In der Schweiz erfolgt die Hepatitis-C-Versorgung der marginalisierten Patientengruppen von Drogenkonsumierenden, falls überhaupt, in erster Linie durch darin spezialisierte Allgemeininternistinnen und -internisten.

Die Versorgungssituation in der Schweiz bezüglich Hepatitis C ist jedoch unbefriedigend, nur 7\% aller Betroffenen wurden in der Schweiz bislang behandelt [5]. Personen mit Drogenkonsum wird die Therapie weitaus weniger häufig angeboten als Leuten, die sich nicht über Drogenkonsum angesteckt haben $[6,7]$. Hauptgrund hierfür ist, dass die diesbezüglichen Angebote der Sekundär- und Tertiärversorgung für viele Drogenpatienten zu hochschwellig sind. Drogenkonsumierende können mit gleich hoher Erfolgsquote gegen Hepatitis C behandelt werden wie Nicht-Drogenkonsumierende [8]. Durch genügend hohe Therapieraten bei Drogenpatienten (auch aktiv konsumierenden) kann die Gesamtprävalenz von Hepatitis $C$ entscheidend gesenkt werden. Die Hepatitis-C-Therapie ist somit ein probates Mittel für die Primärprävention [9].

Engagierte Ärzte bemühen sich aktuell in Zusammenarbeit mit dem Direktionsbereich öffentliche Gesundheit des BAG um ein national koordiniertes Vorgehen zur Verbesserung der Hepatitis-C-Versorgung in der Schweiz.

\section{Limitatio für Verschreibung von Hepatitis-C-Proteaseinhibitoren}

Mit der Zulassung der Hepatitis-C-Proteaseinhibitoren Incivo und Victrelis hat der zuständige Be-

\section{L'économicité à court terme comme principe}

Lorsque I'OFSP a autorisé la mise sur le marché des nouveaux inhibiteurs de la protéase, leur prescription a été limitée aux spécialistes en infectiologie et en gastroentérologie. En y regardant de plus près, cette limitation est contraire aux principes économiques et ne répond pas aux critères d'économicité et d'efficacité exigés par la LAMal. L'épidémie d'hépatite C en Suisse, qui affecte principalement les personnes qui consomment de la drogue, risque de faire exploser les coûts de la santé. La prise en charge médicale est insuffisante et les taux de traitement trop faibles. II s'agit d'empêcher les restrictions qui favorisent cette situation désastreuse et qui n'atteignent pas l'objectif voulu. Afin d'éviter de telles erreurs à l'avenir, il est nécessaire d'impliquer des spécialistes disposant de compétences approfondies dans ce domaine dans la procédure d'autorisation de médicaments pour des structures de soins complexes comme c'est le cas pour le traitement de l'hépatite $C$.

reich des BAG (Direktionsbereich Kranken- und Unfallversicherung) folgende Limitatio ausgesprochen:

Die Verschreibung darf ausschliesslich durch Fachärzte für Gastroenterologie, insbesondere Träger des Schwerpunkttitels Hepatologie, und Fachärzte für Infektiologie erfolgen.

Begründet wird dieser Entscheid mit den Artikeln $24 \mathrm{ff}$ des KVG, die besagen, dass für eine qualitativ hochstehende und zweckmässige gesundheitliche Versorgung zu möglichst günstigen Kosten die zu übernehmenden Leistungen den Kriterien der Wirksamkeit, Zweckmässigkeit und Wirtschaftlichkeit entsprechen müssen. Mit der beschlossenen Limitatio will die Abteilung Krankenversicherung des BAG sicherstellen, dass der Einsatz von Incivo und Victrelis in einem zweckmässigen Rahmen erfolgt. Dazu gehört eine praktikable Überprüfbarkeit der Limitatio durch die Krankenversicherungen. 


\section{Am Ziel vorbei}

\section{Nicht wirksam}

Bei einer gesamtheitlichen Betrachtung der aktuellen Hepatitis-C-Versorgung in der Schweiz kommt man zum Schluss, dass die Verschreibungs-Limitatio von Incivo und Victrelis ihr Ziel nicht nur verfehlt, sondern auch ungewollte Folgen haben wird. Die Verschreibungs-Limitatio für HCV-Proteaseinhibitoren betrifft vor allem Kollegen und Kolleginnen, die sich um die Hepatitis-C-Therapie bei Drogenabhängigen kümmern. Dieser Versorgungspfad ist aber von grosser Bedeutung, da es für viele Hepatitis-CPatienten der einzig gangbare Weg einer Therapie ist. Gerade Drogenpatienten sind häufig nicht in der Lage, Sprechstunden in Spitälern oder spezialisierten Praxen aufzusuchen. Was aber nicht heisst, dass sie nicht erfolgreich und qualitativ hochstehend gegen Hepatitis C therapiert werden können.

Die beschlossene Limitation gefährdet Teile der langjährigen Bemühungen, die Hepatitis-C-Versorgung in der grössten Risikopopulation, den Drogenabhängigen, zu verbessern und so der Epidemie Einhalt zu gebieten. Sie erfüllt somit das Kriterium der Wirksamkeit nicht.

\section{Längerfristig nicht wirtschaftlich}

Bei der Hepatitis-C-Therapie von Drogenpatienten gibt es neben der individuellen Indikation auch eine übergeordnete, epidemiologische: Da sie das grösste Virusreservoir bilden und iv-Drogenkonsum mit Abstand der häufigste Ansteckungsweg ist, gilt es auch mit möglichst hohen Therapieraten in dieser

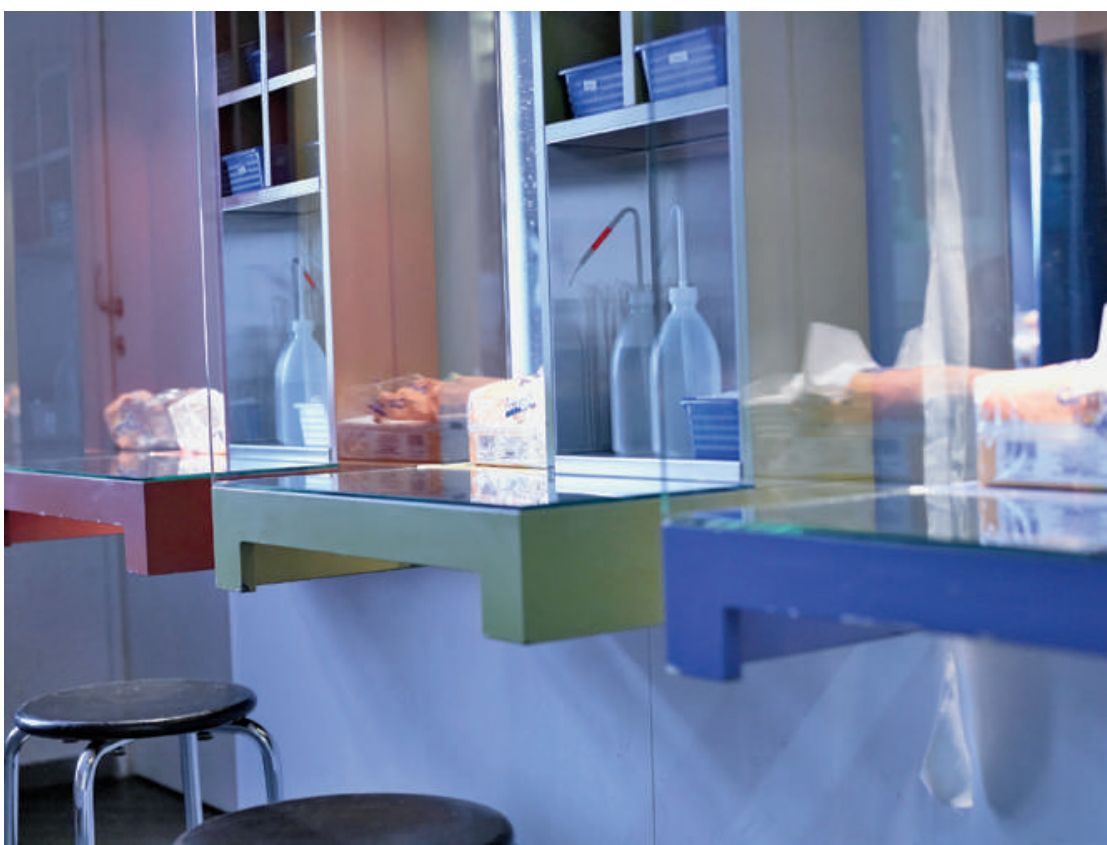

Die Verschreibungs-Limitatio für HCV-Proteaseinhibitoren trifft vor allem Ärzte, die sich um die Hepatitis-C-Therapie bei Drogenabhängigen kümmern.
Gruppe die Prävalenz zu senken und somit Folgekosten zu verhindern.

Diese Limitatio wird verringerte Therapieraten und somit erhöhte Folgekosten von Unbehandelten nach sich ziehen. Das Kriterium der Wirtschaftlichkeit wird aus einer gesamt-gesundheitsökonomischen, längerfristigen Sicht nicht erreicht werden, im Gegenteil.

\section{Zweckmässigkeit nicht gewährleistet}

In der Gruppe der Fachärzte, die von der Limitatio ausgenommen sind, gibt es eine beträchtliche Zahl, die sich nicht mit Hepatitis-C-Therapien beschäftigt und daher keine diesbezügliche Erfahrung aufweist. Mit der Limitatio ist die Zweckmässigkeit somit nicht erfüllt.

\section{Einzig erfülltes Kriterium: Überprüfbarkeit}

Die Limitatio erfüllt hingegen voll und ganz das Kriterium der Überprüfbarkeit für Krankenversicherer. Es kann jedoch aufgrund der oben beschriebenen eingeschränkten Zweckmässigkeit nur die Limitation selber, aber nicht die Qualität der Leistungserbringung überprüft werden. Es fragt sich auch, wie es um die Relevanz dieses Kriteriums steht. Gibt es Grund zur Befürchtung einer unqualifizierten Anwendung eines sehr teuren, komplexen Medikamentes, das nur in Kombination mit einer bisherigen Standard-Zweierkombination (die wohlgemerkt keiner Verschreibungslimitation unterliegt) angewendet werden kann?

\section{Undurchsichtige Argumentation}

Der Direktionsbereich Kranken- und Unfallversicherung des BAG wurde von verschiedener Seite aufgefordert, die besagte Limitation rückgängig zu machen, so auch von den Fachgesellschaften für Infektiologie (SGInf), Gastroenterologie (SGG) und Suchtmedizin (SSAM), von der Expertengruppe für virale Hepatitis (SEVHep), dem Zentralvorstand der FMH und von Hausärzte Schweiz.

Alle diese Begehren wurden vom BAG abgelehnt. In der Argumentation verweist der zuständige Bereich des BAG einerseits darauf, dass Proteaseinhibitoren nur einmal verordnet werden müssen und durch diesen einmaligen Akt die Limitatio nicht kostentreibend sei und das Vertrauensverhältnis zwischen Grundversorger und Patient nicht tangiere. Andererseits wird im gleichen Schreiben das Argument aufgeführt, dass zur Einhaltung der Zweckmässigkeit der Therapieerfolg engmaschig überwacht werden muss und daher nur von den erwähnten Subspezialisten ausgeführt werden soll.

Dieser Widerspruch in der Argumentation lässt den Verdacht aufkommen, dass abgesehen von der Überprüfbarkeit und dem eventuell kurzfristigen positiven Effekt auf die Kosten, der Entscheid zu dieser Limitatio nicht auf einer fundierten Kenntnis der Gesamtsituation beruht. Der Vorschlag, die Limita- 


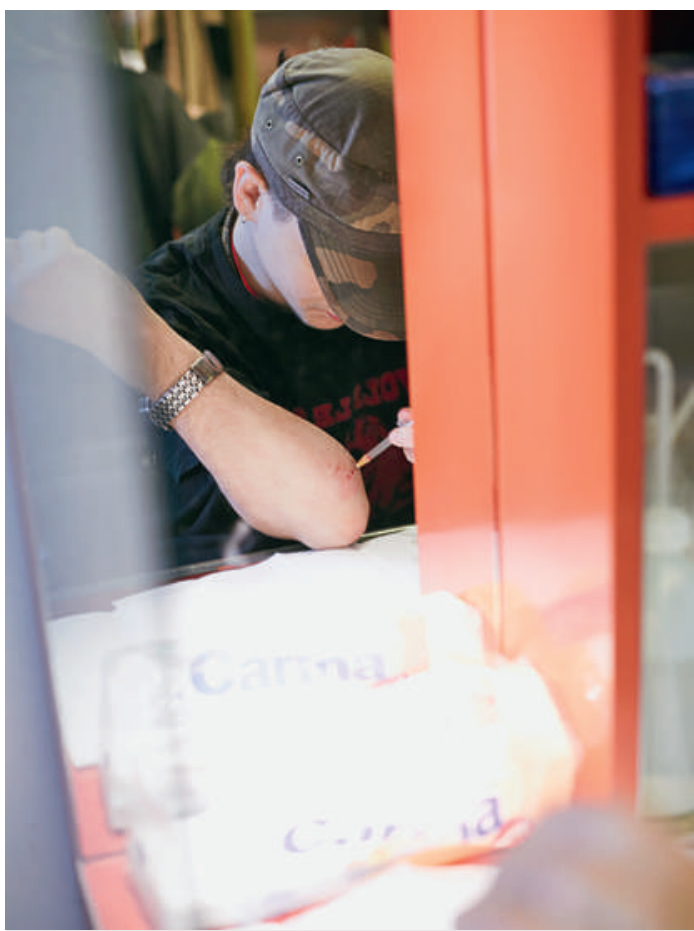

Heroinverschreibungsprogramme - im Bild das Zentrum Stampfenbach der Arud in Zürich - bieten ein ideales Setting für eine in die Basisversorgung integrierte Hepatitis-C-Therapie.

tio analog zum ebenfalls teuren Medikament Xiapex (bei Dupuytren) auf «Ärzte mit Erfahrung in der Hepatitis-C-Therapie» umzuformulieren, wurde mit dem Argument der Überprüfbarkeit abgelehnt. Wiederholte Gesprächsangebote zur Erläuterung der Anliegen und der gesamtheitlichen Sicht wurden vonseiten des Direktionsbereichs Kranken- und Unfallversicherung des BAG ignoriert.

\section{Schlussfolgerung}

Die gesamtheitliche Betrachtung der Verschreibungs-Limitatio der neuen Hepatitis-C-Proteaseinhibitoren bringt die Erkenntnis, dass sie weder zweckmässig noch wirksam und langfristig sogar unwirtschaftlich ist. Im Sinne des angestrebten national koordinierten Vorgehens zur Verbesserung der Hepatitis-Versorgung in der Schweiz ist die diesen Bemü- hungen zuwiderlaufende Limitation rückgängig zu machen. Die Überprüfbarkeit für Krankenversicherer ist von untergeordneter Bedeutung; sie darf in einer Gesamtabwägung nicht entscheidend sein. Die Rolle von Grundversorgern darf nicht weiter geschwächt werden. Der Wegfall von Allgemeinpraktikern mit Spezialisierung in der Hepatitis-Versorgung von Randgruppen wäre mit immensen Mehrkosten verbunden. Nicht zuletzt gilt es, ein Präjudiz für die Zulassung künftiger Hepatitis- und HIV-Medikamente zu verhindern.

\section{Literatur}

1 Ly K, Xing J, Klevens M, Jiles R, Ward J, Holmberg S. The Increasing Burden of Mortality From Viral Hepatitis in the United States Between 1999 and 2007. Ann Intern Med. 2012;156:271-8.

2 Darke S, Kaye S, Duflou J. Systemic disease among cases of fatal opioid toxicity. Addiction. 2006;101(9): 1299-305.

3 Thein H et al. Estimation of Stage-Specific Fibrosis Progression Rates in Chronic Hepatitis C Virus Infection: A Meta-Analysis and Meta-Regression. Hepatology. 2008;48:418-31.

4 Martin NK, Vickerman P, Miners A, Foster GR, Hutchinson SJ, Goldberg DJ, Hickman M. Cost-effectiveness of hepatitis $\mathrm{C}$ virus antiviral treatment for injection drug user populations. Hepatology. 2012;55(1):49-57.

5 Lettmeier B, Mühlberger N, Schwarzer R, Sroczynski G, Wright D, Zeuzem S, Siebert U. Market uptake of new antiviral drugs for the treatment of hepatitis C. J Hepatol. 2008 Oct;49(4):528-36.

6 Witteck A, Schmid P, Hensel-Koch K, Thurnheer MC, Bruggmann P, Vernazza P; Swiss Hepatitis C and HIV Cohort Studies. Management of hepatitis $\mathrm{C}$ virus (HCV) infection in drug substitution programs. Swiss Med Wkly. 2011;27:141.

7 Schulthess K. Versorgungsqualität von opioidsubstituierten Patienten mit chronischer Hepatitis C - Eine Querschnitt-Studie im Kanton Zürich. Inauguraldissertation Med. Fakultät der Universität Zürich; 2011.

8 Hellard M, Sacks-Davis R, Gold J: Hepatitis C treatment for injection drug users: a review of the available evidence: CID. 2009;49:561-73.

9 Martin $\mathrm{N}$ et al. Can antiviral therapy for hepatitis C reduce the prevalence of HCV among injecting drug user populations? A modeling analysis of its prevention utility. J Hepat 2011;54:1137-44. 